
\title{
$\begin{array}{ll}\text { Research Square } & \begin{array}{l}\text { They should not be considered conclusive, used to inform clinical practice, } \\ \text { or referenced by the media as validated information. }\end{array}\end{array}$ \\ Corpus Driven Machine Translation Framework for Students
}

\section{Zhengxu PAN}

Jilin International Studies University

Lei Wang ( $\nabla$ wqsubrina@126.com )

Changchun Sci-Tech University

\section{Research Article}

Keywords: Corpus Training, Machine Translation, Decoding, Students, Learning

Posted Date: October 26th, 2021

DOl: https://doi.org/10.21203/rs.3.rs-945493/v1

License: (c) (i) This work is licensed under a Creative Commons Attribution 4.0 International License. Read Full License 


\section{Corpus Driven Machine Translation Framework for Students}

Zhengxu PAN' ${ }^{1}$, Lei WANG ${ }^{*}$

ISchool of Oriental Languages, Jilin International Studies University,

Changchun 130117, Jilin, China

2School of Language and Culture, Changchun Sci-Tech University,

Changchun 130117, Jilin, China

1panzhengxu1979@126.com

2wqsubrina@126.com

\section{Abstract}

Machine translation can be used as a language-based method, where words are translated into the most appropriate language where they will be replaced. Students are willing to automatically learn knowledge interpretation from large data instead of writing rules on human professionals. Although the end-to-end machine translation (MT) process has recently made considerable progress, the problem of low-resource language pairs and areas still suffers from data scarcity. In this paper, an architectural statistics source-based translation machine (ASS-TM) model has been introduced to deal with the data scarcity problem need to translate small body language. The discriminative learning process (DLP) is employed to enlarge the vocabulary of a system and set of syntactic structures by integrating the synonyms and paraphrases obtained in corpus training. The iteration pipelines for the integration and combination of various generation models using an effective decoding framework. Symmetric context-free grammar (SCG)is implemented to extract a translation memory that includes the conceptual relationships between the two component's structures. The simulation analysis is performed based on accuracy and efficiency, proving the proposed framework's reliability of $97.3 \%$.

Keywords: Corpus Training, Machine Translation, Decoding, Students, Learning

\section{Introduction to corpus-driven machine translation}


MT is a basic translation pattern with the advantage of converting large text areas within a concise period [1]. Many composite translation systems have succeeded in improving translation accuracy [2]. Currently, hybrid systems with the most commonly used MT systems are based on the rules and facts. Several research projects combining both methodologies have been carried out [3]. Machine translations are best used for the overall idea and the core of an enactment or content [4]. However, in terms of phrase accuracy, a machine will not deliver a high correct translation from one language to another [5].

Machine translation based on corpus is a set of reference conversions from which a new text is translated, is used by corpus systems [6]. With machine translation in a corpus, a training phase of the system is assumed to have equivalence significances [7]. The Information Technology (IT) Revolution and technological developments have contributed to the growth and expansion of communicative industries [8]. With numerous new techniques and specifications, the use of machine translation has grown unparalleled [9]. However, in a web environment, the major focus of researchers in business is to develop effective and precise translation systems quickly and the total number of translations available in different languages [10]. This scientific use of corpus for special machine pronunciation and computerized translation applications is involved [11]. With the progress in storage and computing capabilities and the acquirement of massive quantities of text, the company's use of multilingual concurrent text seems a promising tool for the long term [12].

This method is based on the fact that no predefined interpretation solutions are available; however, possible solutions are already available in texts transformed by experts [13]. In other statements, the linguistical equivalence found in already translated texts codes a large part of the skills of a translator [14]. Moreover, a multilingual corpus is richer in language information than a bilingual corpus since it provides information on situational 
equivalence on the potential of the particular language when in contact with a language system differently [15].

\subsection{Machine translation framework for students}

Translations can help students learn to write their mother tongue without using a different language [16]. For instance, the teachers may require students to 'transfer' an academic document into a more conversational text or vice versa [17]. Applications for machine learning (ML) are widely applied today, and language teaching is one of them [18]. Machine translation (MT) does not help learners to translate for everyone there [19]. They are available and can be used easily on most websites, such as Google Translates [20].

The purpose of this study has been to demonstrate the role of machine translation in improving translation skills and the influence of MT on efficient learning [21]. It is fully automated and removed from human interference; it has been used in most areas [22]. Many artificial intelligence applications have emerged with the renewal of advanced technologies such as picture recognition, natural language processing, interpretation, healthcare, and even robotic systems [23]. Translations based on machines and second language innovations were stimulated by technologies and the widespread use of computers in schools [24]. Therefore, the objective of this study is to investigate and make an enjoyable and interactive translation software in terms of language skills and conversion [25].

The technical innovations of this research involve,

$\checkmark$ An architectural statistics source-based translation machine is implemented to improve the availability of data for the learning process.

$\checkmark$ The discriminative learning process (DLP) is applied to enhance the language skills of a framework.

$\checkmark$ Symmetric context-free grammar is enacted to retrieve a translation memory covering the complex connections between the structural parts. 
The paper is structured as follows: Section I describes the importance of machine translation in the learning process and introduces a corpus-driven framework, followed by a detailed literature review in Section II. A complicated mathematical equation of an architectural statistics sourcebased translation machine (ASS-TM) model is concerned with section III. The experimental outcomes were demonstrated in section IV. Finally, the paper concludes with section $\mathrm{V}$.

\section{Literature survey on traditional corpus-driven translation techniques}

It has an impact on the translation industry, which further exchanges and manages information. Therefore, this study explores the future role of machine translation and interactive activity in learning the English language and translation. Thus, different studies in translation in the corpus-driven framework are carried out, particularly in $M L$ applications and their significance for education.

Wu J. et al. [26] aimed to provide communicative students with education using web-based machine translation (WBMT) tools through learner assessments and attitudes. In particular, the level of dependence of students and the WBMT value for language purposes are assessed. This study introduced machine translation experimental classrooms, implemented by the MT study sites, with 45 students at Silesia. The output of the MT was collected based on testing students with knowledge and understanding of MT techniques.

Way A. et al. [27] examined how teacher educators view mathematical notation in a highly technologically friendly university environment and how it affects students' personal educational experiences. It raised how introducing new instruments could disrupt the link between various elements in an experimental platform. They focused on the quality of the comment product compared to the significance of human translation(CPCS-HT). The effectiveness was inferior to the state of human translation based on attitudes towards machine translation. 
It has drawn the history to its advancement and considers its beginning with early stages. In the past several years, the field of MT on web has offered an overview of two key topics and the most considerable input in literature. The role of these online MT tools in meeting the user's textual needs and their impact on the business and the economy was evaluated byMTKahlon N. K. et al. [28]. Artificial Intelligence applications need to overcome significant implications in our daily lives., English reading comprehension using artificial intelligence (ERCA-AI) has been introduced to enhance this. Technology has always been seen as a great system of support in language learning.

Aksoy B. N. et al. [29] presented Parse and Corpus-based translation(PaCo-MT), a data-based approach to probability syntax machine translation based on rules. Tree-based data-driven system-induced translation rules instantaneously from a large syntactic investigated parallel corpus, created in a different concept: the phrase-based statistical machine translation systems, strings-based and ground rules use any language knowledge. This architecture was detailed and an assessment compared to our earlier work and the modern machine translation system.

Luqman $\mathrm{H}$. et al. [30] suggested that the translation should empower a $P C$ in semantic, syntactic, and textual measurements to form a characteristic language (SST-CL). Moving a word or speech from one language sometimes can be powerfully carried out with MT, and it might have evident and unexpected implications. In other words, it is starting to write, and a machine cannot disintegrate culture requirements in the contents of texts and speech. Because of the two opposing views, our study found how these two can be combined and which advantage can be obtained.

However, these studies did not specify the use of MT explicitly in interpretation education and learning. They guided the use of MT as an ineffective tool or an additional tool in class. This involves comparing existing methods like WBMT, CPCS-HT, PaCo-MT, ERCA-Al, and SST-CL with ASS-TM's proposed method. 


\section{Implementation of architectural statistics source-based translation machine ASS-TM}

The following approach depends greatly on the linguistic environment. Therefore, it involves intensive use of resources in terms of work and hours to create regulations. It is difficult to implement or easily extended to other languages and is given with mathematical expressions.

\subsection{Source-based translation machine}

The unidirectional bilingual machine translation called word-based or dictionary-based translation usually requires structural analysis of source text to achieve the requirement of basic linguistics.

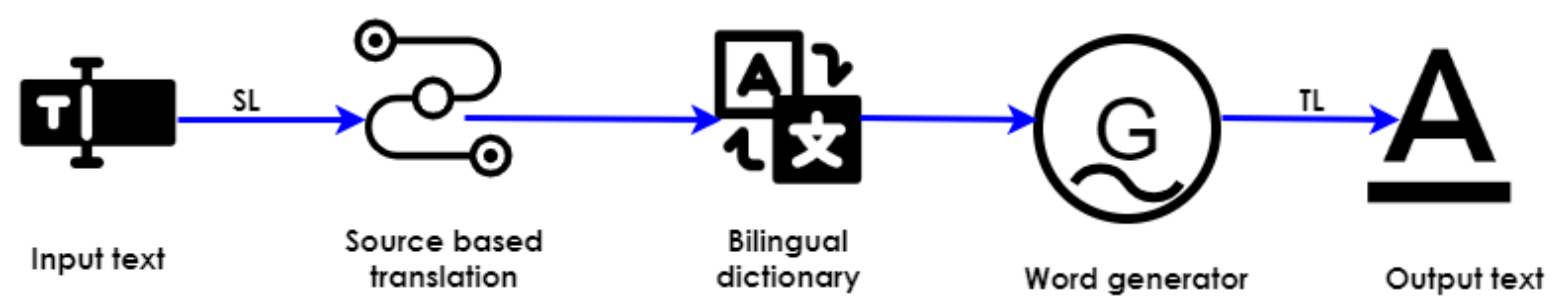

Figure 1: Framework source-based translation machine

The morphologic analyzer's level or quality, bilingual translations, and conformational changes rules establish system performance. Moreover, direct systems are susceptible to lexical errors and inappropriate syntactic structures closely related to source language(SL), as shown in Figure 1.

\subsubsection{Text and output input}

MT replaces words in one language by input texts given differently; however, this is rarely the way good translation is produced. It is necessary to identify whole sentences, and their closest counters in the target language are the output text.

\subsubsection{Source-based translation}

A source text is a translation that derives knowledge and ideas. The original wording is to be transposed into some other language as a source text in translation. In the practice of translation, the source language is the vocabulary from which the language is translated to the content knowledge known as the receptor language. 


\subsubsection{Bilingual dictionary}

The interpretation into another format of the natural language processing written in one machine translation is bilingual translation software. The computer automatically translates the paragraph, which supplies text translations without user intervention based on computer algorithms. The source language is rapidly and effortlessly translated into one or more target languages with MT.

\subsubsection{Word generator}

Structural analysis is done for each SL input text, after which it transformed to an intermediate image is mainly made by the abstract parse tree. The destination text is obtained by structural converting based on the specific generator, according to figure 1. The analysis of this text is done in SL structural analysis. The following sub-section elaborates SL and the target language (TL).

\subsubsection{SL and TL}

In translation practice, the source language is converted to vocabulary from which it is translated. Whereas the target language is known as the receiver language, is again inversely translated. The source language $(S L)$ is often the primary language of any translator and the target language $(\mathrm{TL})$.

$$
\begin{aligned}
& f f(x+y)=\iint f f(x)+f f(y) * \int_{i=x}^{y} f f x(y) * f f y(x) \\
& \frac{d(f f)}{d x d y}=\int_{i=1}^{x} \int_{i=1}^{y} \frac{\partial(d x d y)}{\partial^{2}} \\
& d^{n}(f f)=\sum_{a=0}^{N}\left(\begin{array}{l}
N \\
a
\end{array}\right) d^{n}(f f(x)+f f(y))^{N-1}
\end{aligned}
$$

Operators for grammar over a word are analyzed in source-based translation grammar with formal functions $f f$ are stated in the interrelation equations 1,2, and3. As phrases taken for examples $x, y$ are independent with the translation variable $i$ for the double integral functions of limits $i=x$ to $y$ used here. Differential functions $\frac{d(f f)}{d x d y}$ in the source-based translation with the certain limits of $i=1$ to $x$ and $i=1$ to $y$ for the separate phrases can be 
considered as an abstract constant symbol $\partial$ for a variable $d^{n}$ capability. In regularly to secure structured functions with summation process of same limits with some translation $N$ involved contain common difference rule afor the derivative of a letter in $N-1$ could be defined as a formal framework with $a=0$ to $N$.

Machine translation is a computerized system that translates text into a basic language known as the source language (SL) in another human language, called the target language (TL), with involvement in regaining the meaning of the original text into a natural language

\subsection{Translation model}

Translation based on a rule is mostly used for multilingual translation, and two approaches, transfer and interlinks belonging to the second generation of machine translation are possible here. There are three main stages of two associated representations, one relating to $\mathrm{SL}$ and the other relating to $\mathrm{TL}$ : evaluation, process, and formulation, described below.

Source language

Target language

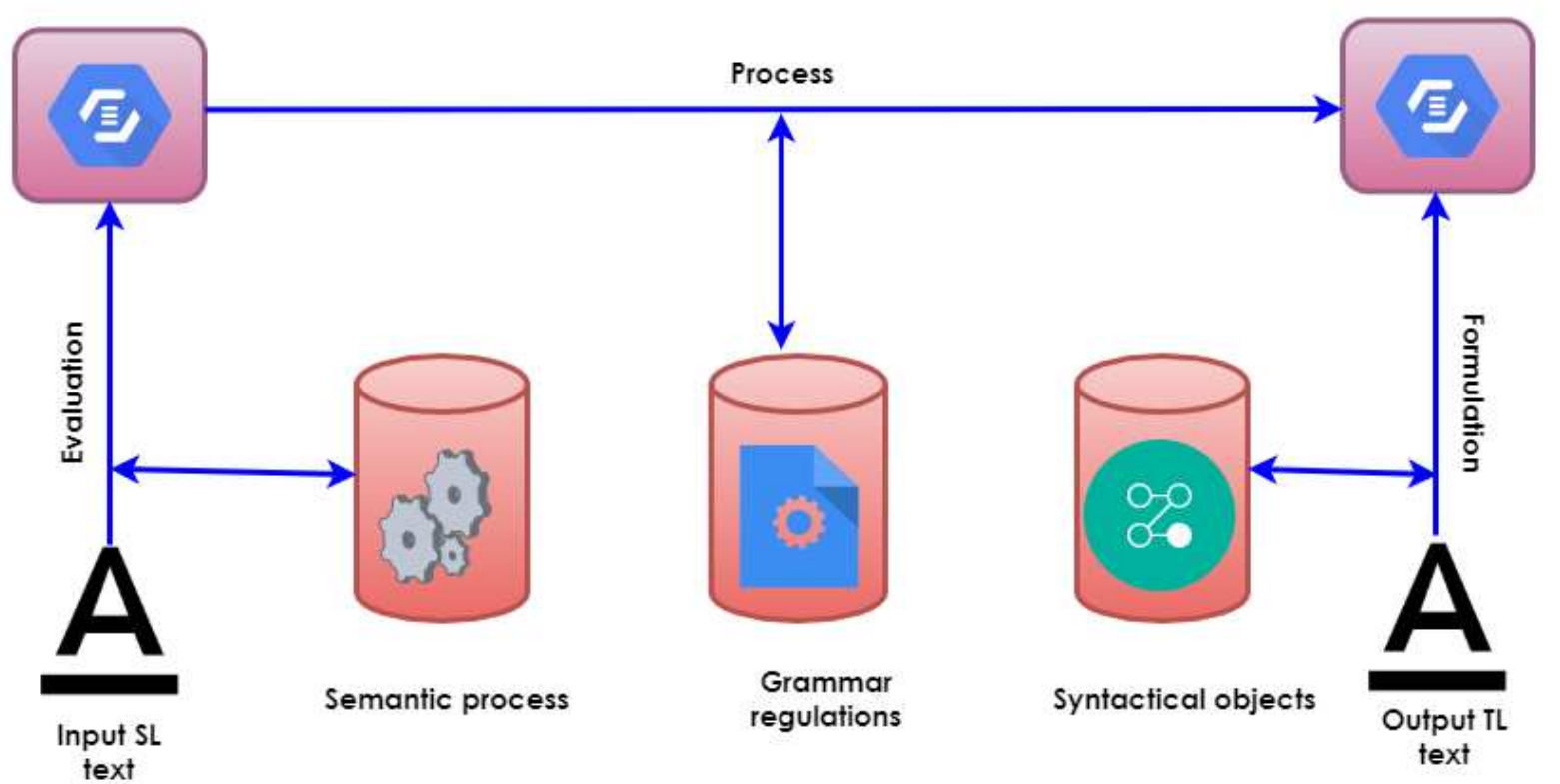

Figure 2: Process of translation model

In terms of the morphological characteristics, analysis of each sentence structure and semantics process in the evaluation stage of the source language text is expressed in figure 2. The morphology includes identifying the basic set of words; component of the speech, spelling and 
punctuation removal, the privacy policies involves the creation of phrase structures, lexical linkage, etc. In semantic and syntactical objects creation, algorithms or analytical approaches can be used.

IT is a source-language, abstract intermediate, and uses SL-related vocabulary that includes morphological characteristics, grammatical and semantic structures. The transfer phase involves converting the transitional language related to SL to $\mathrm{TL}$ using a lexical database with grammar regulations governing the basic SL and TL formulations. At the synthesis phase, a compatible structural and semantic shape (semantics), correct word aspects (morphology), and the appropriate statement or grammatical structures are created. A dictionary that has TL structures, function, and semantic structures is necessary.

$\left\{\begin{array}{l}l_{a}=G_{a} \rightarrow l_{\sigma}\left(S_{i}\right) l_{\delta} \\ l_{b}=G_{b} \rightarrow l_{\sigma}\left(S_{j}\right) l_{\delta}\end{array}\right.$

$\left|\frac{p_{1}}{P_{1}}-\frac{p_{2}}{P_{2}}\right| \leq \sqrt{\frac{\sin l_{\sigma}\left(S_{i}\right) * l_{\delta}}{2 * \frac{p_{1} p_{2}}{P_{1}+P_{2}}}}$

$d t\left(P_{1}, P_{2}, p_{1}, p_{2}\right)=\frac{p_{1} p_{2}}{P_{1}+P_{2}} *\left(\frac{p_{1}}{P_{1}}-\frac{p_{2}}{P_{2}}\right)^{2}$

The proposed system will combine the corresponding TL structure; the sequence $S_{i} \approx S_{j}$ implies the merging of all pairs. The lexical database $l_{a}, l_{b}$ with corresponding constant $l_{\sigma}$ and $l_{\delta}$ are denoted as the product of the analyzer. The regulations of the grammar involved are $G_{a}$ and $G_{b}$ are defined in equation 4 . Two proportions $p_{1}, P_{1}$ and $p_{2}, P_{2}$ are not statistically different when using this test for proportion comparison in the translation model. Here, the communication dissimilarity between two proportions with the square root of products is multiplied with sin in equation 5.Therefore, for all contexts of different tests carried out according to the variables as described in equation 6 , the differences of the two parameters are achieved as a maximum value for $d t$. 
If $n$ languages are used, the transaction subsystem pair $n(n-1)$ is necessary, which leads to the interconnectedness of convenient way of collecting times in system design.2n pair of components are needed for this $n$ number of language using this model to achieve correlation coefficient.

\subsection{Corpus framework with a machine translation}

The key concern in developing an MT system for SLS is the compilation of the bilingual corpus. As the SLMT system without a processing or corpus configuration does not have an appropriate, standard corpus, a bilingual corpus, and therefore, these must be developed in this study.

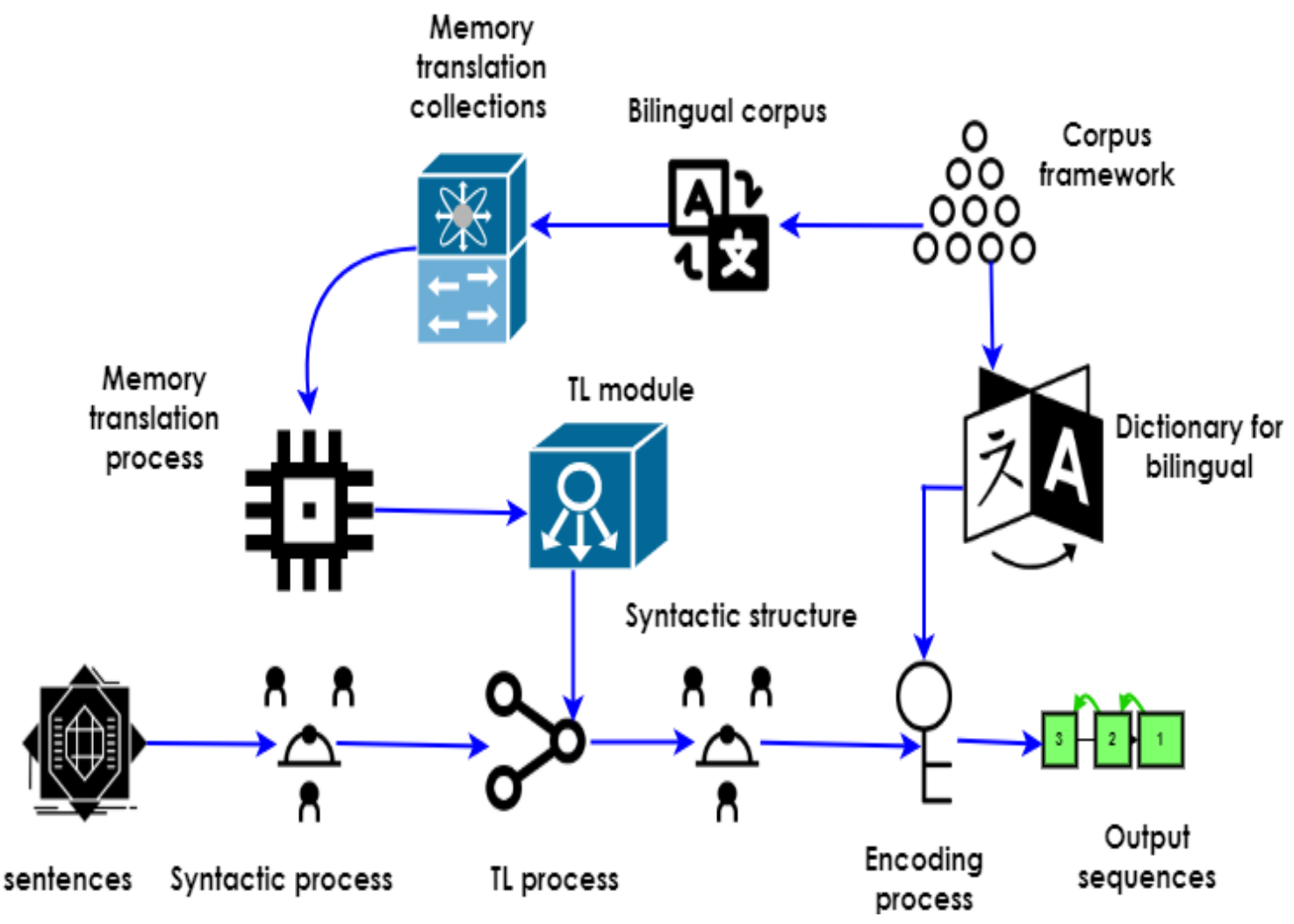

Figure 3: Proposed corpus framework with a machine translation

This article investigated a statistics source-based translation machine (SSTM) model for the scarcity of data using a simple corpus by translation process from the input sentences. The syntactic process in the proposed framework is illustrated in figure 3. The developed TL process and formatted parallel bilingual corpus with a symbol representing the reasons related to a sign and a format usually in a dominant language with encoding process 
having the same meaning. The syntactic process memory of linguistics consists of the conceptual relation of the two components' grammatical rules. The SSTM framework has been used to associate the conceptual roles in the grammatical structures for the memory translation process. The translation memory collections provide the TSL structure of transcription comparison frameworks. Finally, the TSL contract documentation included in the bilingual corpus dictionary is used to interpret the TSL sequence to match the information on an arrangement in the corpus framework. The development process and the translation memory retrieval from the bilingual corpus will occur in the translation module. The structural MT statistical model that combines the statistical procedures and the translation recollection to interpret syntax structures is hypothesized to generate output sequences.

$$
\begin{aligned}
& \delta_{l \rightarrow d}=\arg \max \left\{\sum_{i=1}^{l} \sum_{j=1}^{d} \log (s l \mid s d)_{l \rightarrow d}\right\} \\
& d_{l \rightarrow d}=\arg \max \{p(s l \mid s d)\} \\
& d_{l \rightarrow d} ; l_{l \rightarrow d}=\frac{\arg \max \left\{p_{1}(s l \mid s d)\right\} *\left\{p_{2}(s l \mid s d)\right\}}{2 * p_{1}+p_{2}}
\end{aligned}
$$

Machine translation treats simultaneous corpus as the main source of translation knowledge as a data-driven approach. Let $s l$ be a sentence of a linguistic system $l$ and $s d$ be a statement of destination $d$ interlink process of using $l \rightarrow d$ denote a cognitive linguistic model component, where fixed to files in a set of model parameters $i, j$ for the summation limits of $\arg \max$ of $i=1$ to $l$ and $j=1$ to $d$ inclined in equation 7.The probability function of the translation needs to be done with $p$ and given as $\{p(s l \mid s d)\}$ in equation 8.The average probability of $p_{1}, p_{2}$ with the $\arg \max$ are denoted in equation 9.

However, researchers have to translate the TL parallel sequence data manually; there will be a limited amount of bilingual sentence pairs. This section analyzes existing methods for developing a bilingual body and extracting translation memories for the source language model. In addition, the conceptual roles can be used to reduce grammar alterations with the extract meaningful grammatical rules of the same signification. 


\subsection{Discriminative learning process}

Discriminatory algorithms of the learning process have helped to improve the performance of natural language parallelization and labeling. The algorithms of discriminative processing for these implementations include convolution, enhancing, and vector support decisions. Some new discriminatory classifications were proposed in recent years in the machine-learning society.

\section{Algorithm 1}

\section{A discriminative learning algorithm for machine translation framework}

Initialize $t, b$, learning process index $X$

$t \leftarrow 0$, assigning $V^{0}$

\section{repeat}

for $(i=0 ; i>=; i++)$ do

Process $V^{0}$ with index value $X$

for $(j=1 ; j>=0 ; j++)$ do

Compare (value $t$ with $i$ )

Update index value

Increment index $i$

else

Update vector $V^{0}$ with index value $X$

Increment index $j$

check index vector value

print the processed words

end for

end for

until no process in outer loop

Algorithm 1 given above is used to partition the unprocessed data by the method of machine translation. However, the upgrade is not conducted until the pairs in one sentence have been found to accelerate the algorithm. Here, first computing the outer loop by initializing the sentences with vector values, assigned with top and bottom values $t, b$ respectively, whereas the 
vector $V^{0}$ is processed with two positive integer values $i, j$. By the index value, processing one loop by checking the integer value and translate the sentence. Then the next inner for loop can be processed with increment index values. Check whether the index value satisfies the processing, print the processed words. Otherwise, repeat the process until all the sentences are translated, and then stop the processing. The process involved through this algorithm is analyzed by the mathematical equation, which is stated as,

$$
\begin{aligned}
& \widehat{c s}=\arg \max _{y} \sum_{x=1}^{c s} c s\left(T s t_{x}, S s t_{y}\right) \\
& c s(T s t, S s t)=p t(l s, l t) * \frac{a c(x, y, l s, l t)}{\sqrt{p t(l s, l t)}} \\
& A v(\widehat{c s}, c s)=\sum_{x=1}^{l s} \sum_{y=1}^{l t} \frac{c s\left(T s t_{x}, S s t_{y}\right)}{\frac{a c(x, y, l s, l t)}{\sqrt{p t(l s, l t)}}} \\
& \operatorname{sim}(\widehat{c s}, c s)=\frac{(T s t|| S s t)}{\sqrt{T s t U S s t}} * A v(\widehat{c s}, c s)
\end{aligned}
$$

For determining the coefficients $c s$ from a source sentence Sst $=$ $\left\{\mathrm{st}_{1}, \mathrm{st}_{2}, \mathrm{st}_{3} \ldots, \mathrm{st}_{\mathrm{n}}\right\}$ to a target sign sequence $\mathrm{Tst}=\left\{\mathrm{ts}_{1}, \mathrm{ts}_{2}, \mathrm{ts}_{3} \ldots, \mathrm{ts}_{\mathrm{n}}\right\}$, the probabilities of finding coefficient for all the words in the corpus-driven framework are processed with arg max with the variables of $y, x$ using the summation process is estimated in the above equation 10. The probability functions $p t$ betweenSst and Tst can be sequenced with actual length ac of source $l s$, and target $l t$ is calculated with the square root functions mentioned in equation 11. The average values $A v$ of both vector-based $\widehat{c} s, c s$ are summed with sequence limits $x=1$ to $l s$, and $y=1$ to $l$ are proposed in equation 12. Finally, similarity $\operatorname{sim}$ between these $\widehat{c s}, c s$ are defined in the equation 13 are calculated with logical $\|$ and $U$ for both $S s t$, Tst. The similarity value achieved with the root value function is always inversely proportional to the logically obtained value.

The above algorithm results slightly better than the baseline and best feature set and the average error preparation with retrieval algorithm on the usable configurations. The differences in the value obtained with calculations 
performed, however, are important. Development data can enhance regression test performance. The theoretical analysis guarantees this property and supports the experimental results.

\subsection{Symmetric context-free grammar (SCG)}

A context-free grammatical $\mathrm{gr}$ is defined over a letter $\mathrm{z}$ as a set of replacement rules that substitute a structured feature in z with lettering. A rule is often called a performance, as informal language theory in error-free grammar. Therefore, a letter in $\mathrm{z}$ could be based on a comprehensive function to ensure simultaneous differential rules cover formal functions.

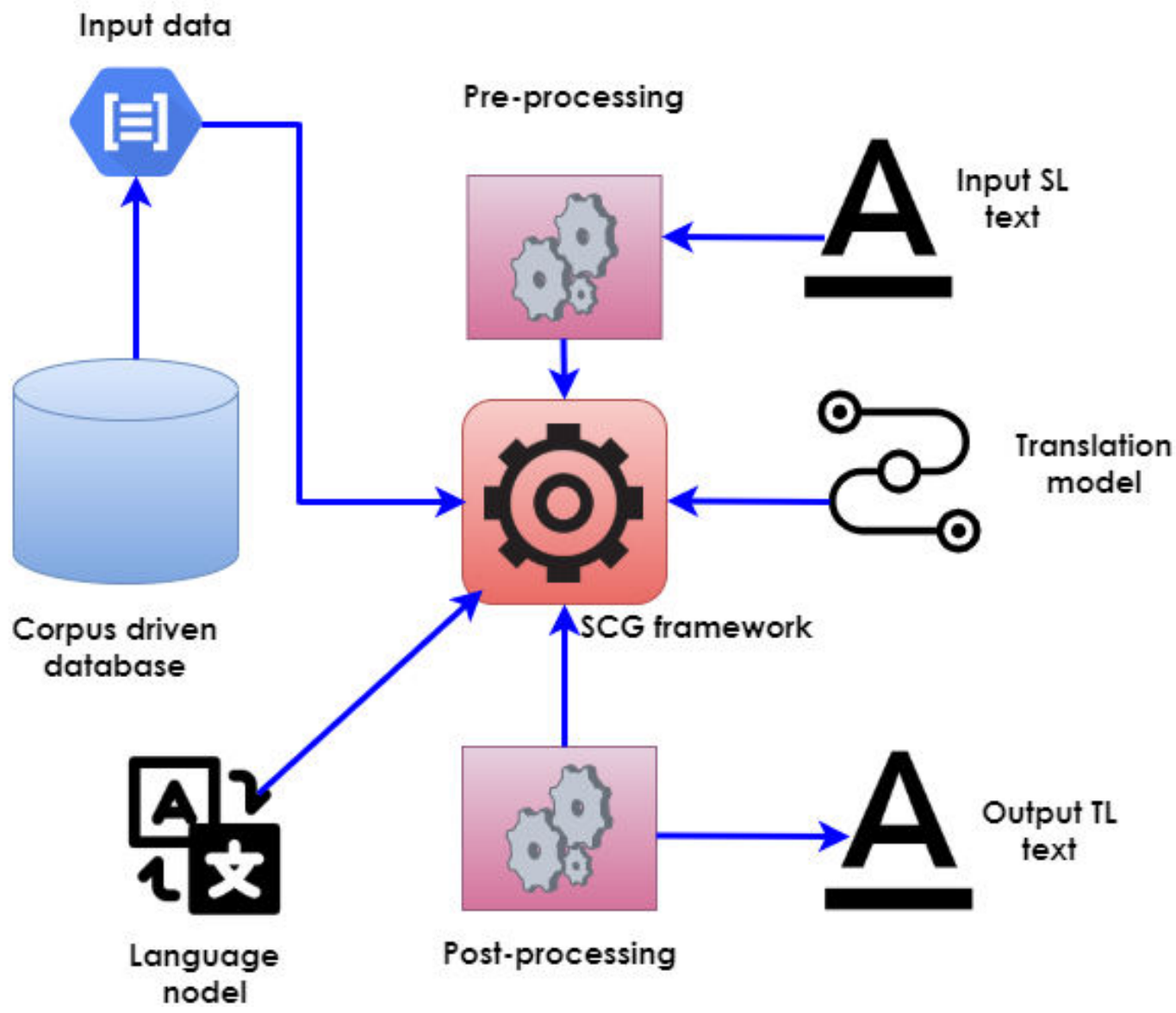

Figure 4(a): SCG framework

Grammatical parsing maps a sentence into a formal specification in natural language. Previous studies have shown that symmetry with contextfree grammar is good, with most alternative options shown in Figure 4(a).

\subsubsection{Corpus driven database}


A corpus is an electronic collection of text pieces selected by empirical evidence as a data source for language teaching to represent as far as possible a language or vocabulary variety.

\subsubsection{Pre and post-processing}

Before an item or access is saved, both pre-processing and postprocessing commands are running. The difference is that before the valueand-validation rules are checked and after these processes, post-processing commands execute.

\subsubsection{SCG framework}

A context-free symmetric is a programming language created using context-free grammar in the written language theory. These languages are often used in computer languages, especially with most arithmetic expressions generated through context-free grammars. A proper sentence structure is "free of context" when manufacturing rules are applicable regardless of a non-terminal context. No matter what symbols are on the upper left side, the single non-terminal on the right side can all be supplemented. This is the difference between a context- grammar and symmetric grammar framework through the input data.

\subsubsection{SL input and TL output text}

Translation includes understanding the equal meaning in the target language and taking into account values of the target language (TL) and source language (SL), regardless of grammatical, contextual, and even linguistic values. The conversion of the process of source into the target language is shown below. 


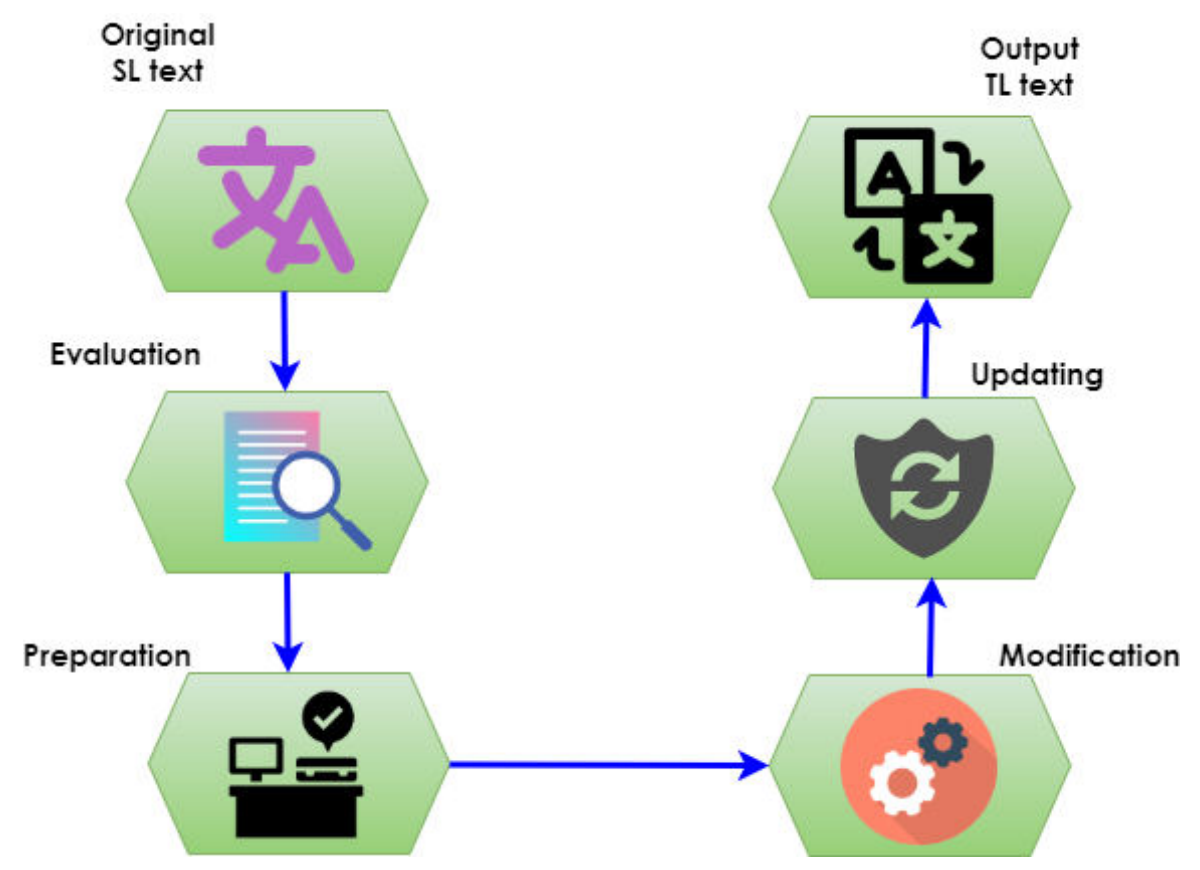

Figure 4(b): Conversion of SL into TL

Translators must practice their translation skills which can be analyzed with various techniques, strategies, and even challenges. Since the translator cannot handle the skills in translation fully, the problem with translating appears to be quite difficult to solve are described in figure 4(b). The right translation deals with how the interpreter may transfer the significance from source to target and how translation source language (SL) text can be produced conventionally. Translation evaluation of the source language directly into the target language includes indebtedness, quantification, and conversion of symbolic content. Updating appropriate translation elements include rearrangement, amplification, appropriateness, and modification. Transposition includes changing the meaning of a message from one-word class to another. The modification itself refers to variations in message form, and the equivalence of the output target language (TL) text in different design and thematic ways are obtained.

\subsubsection{Translation model}

The translation is a process that seeks to communicate from one language to another the definition or meaning of a given language discussion. In terms of the uniformity for meaning across language groups, translation may be characterized. 


\subsubsection{Language model}

The language model explains differentiating between rational unified terms and expressions. Modeling languages are used to recognize the voice, language processing, part-of-speech tagging, pre-processing, text categorization, lettering, information recovery, and other implementations.

$$
\begin{aligned}
& P(i t)=\sum_{p s, i} \frac{\log (n t(p s, i)) / \log (n t)}{|p s|} \\
& \operatorname{dsim}(i t)=\frac{A(p s, i)+R(p s, i)+C(p s, i)}{N(A * R * C)}
\end{aligned}
$$

Here, criteria were introduced in an attempt to discover the appropriate sources to solve these difficulties with several inputs sentences are analyzed as $N$, to use every example as a document probability $P(i t) . n t$ denotes the average number in the bilingual corpus of illustrations. The $p s, i$ word exists the duration is 1.0 therwise the value 0 is combined with the total input $n t$ phrase length and standardized in equation 14. The dissimilarity dsim of sequence in the sentences involves adding $A$, removing $R$, and compensation $C$ of each input sentence's $\log (n t)$ are defined in equation 15.

Machine translation analysis is the development of combinatorial optimization, in short, computational linguistics. The language model can be predicted is taken as a dependent variable with the words preceding it. The modeling language is a core issue for a wide range of data processing in natural languages. For a task that requires linguistic understanding, word embedding is more practical at the front or back end of a sophisticated model.

\subsection{Effective decoding framework}

The effectiveness of any statistical machine translation system is based on a good encryption scheme. The task of the decoder is to find the most likely translation-based prior knowledge parameters and a formula for incorporating them.

$$
P(t a)=\frac{\mathrm{e}(w t(i s) * h t(i s))}{\sum e\left(\frac{w t}{h t}\right)}
$$




$$
\begin{aligned}
& h t^{n}=f n\left(h t^{n-1}\right) * w t \\
& P\left(h t^{n}\right)=\prod_{i=1}^{t a} P\left(h t^{n}\right), \text { where } h t^{n} \in\left(h t \mid h t_{-1}, \ldots, h t_{n}\right)
\end{aligned}
$$

A translation network tacan be appropriately used by teaching about allocation in the next input sequence is of varying length probability $P(t a)$. For example, in the event of a sequence, an encoding translation with productivity can learn the dissemination process said to be e. For all symbols, the possible weight matrix wtfor the sequence is is denoted with hidden state $h t$ function results are shown in the above equations 16, 17, and 18. The number of input sequence is for the hidden state $h t^{n}$ are processed with the weight matrix function $f n$. The value of a pi product with the certain limit for variable $i$ given with the limit value $i=1$ to tafor the probability of hidden state $P\left(h t^{n}\right)$.

It compares acceleration and production efficiency with added decoder's functionalities as a fast greedy decoder and a slow process though optimum decoder that treats decoding as an optimal probation enhancement for integrated coding.

An architectural statistics source-based translation machine (ASS-TM) model in the statistical machine translation (SMT) method is used for structural translation to align the conceptual functions in grammar rules. The linguistic memory provides structure translation frameworks. Finally, the verbs arrangement information has been labeled to enrich translated SMT sequence expressiveness. Several experiments have been carried out to assess the efficiency of source language and interaction.

\section{Results and discussions}

The input grammatical rules are translated utilizing synchronization and translation memory into SL sentence structure. By optimizing experimental work and analyzing the performance of this proposed approach as-TM, the optimal translation TSL sequence is obtained.

\subsection{Analysis of bilingual corpus and extracted memory}


In the semantics TSL translated version, the bilingual dataset usually plays an important part. Analyzing the availability of the extracted translation memory for the source language is performed to assess the performance of the approach proposed ASS-TM. The grammar rules had thematic roles and were taken as fundamental units in the memory of the translation.

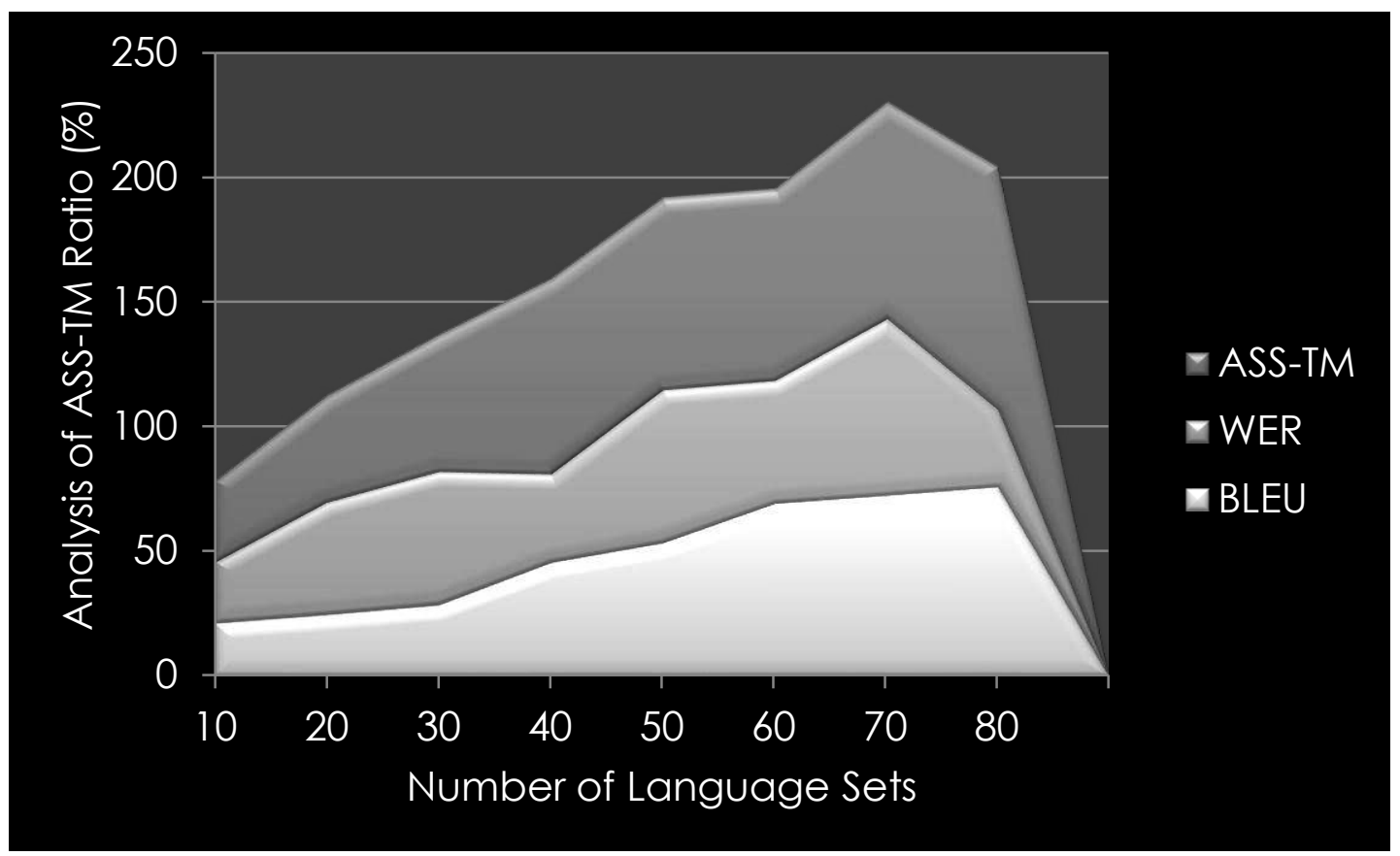

Figure 5: Analysis of ASS-TM with a bilingual corpus

The bilingual collection includes these grammar rules, and this approach can translate the input phrases into corresponding TSL phrases easily and accurately, as shown in figure 5. Here, ASS-TM performance is compared with two lingual, traditional methods word error rate (WER) and Bilingual Evaluation Understudy (BLEU) is applied using the equation (3).

\subsection{Evaluation of TL with grammar rules}

Table 1 Grammar rules evaluation with ASS-IM

\begin{tabular}{|c|c|c|c|c|c|}
\hline Criteria & Subjects & $\begin{array}{c}\text { Phrases } \\
\text { with verb }\end{array}$ & $\begin{array}{c}\text { Phrases with } \\
\text { preposition }\end{array}$ & $\begin{array}{c}\text { Phrases } \\
\text { with noun }\end{array}$ & $\begin{array}{c}\text { Average } \\
\text { (\%) }\end{array}$ \\
\hline $\begin{array}{c}\text { Short } \\
\text { sentences }\end{array}$ & 30 & 45 & 41 & 68 & 70.0 \\
\hline $\begin{array}{c}\text { Long } \\
\text { sentences }\end{array}$ & 34 & 54 & 43 & 73 & 70.9 \\
\hline
\end{tabular}




\begin{tabular}{|c|c|c|c|c|c|}
\hline Baseline system & 37 & 65 & 49 & 79 & 77.6 \\
\hline IBM model2 & 39 & 69 & 52 & 82 & 86.5 \\
\hline WER & 45 & 70 & 56 & 85 & 87.4 \\
\hline BLEU & 43 & 72 & 59 & 85 & 90.1 \\
\hline ASS-TM & 65 & 76 & 67 & 88 & 94.1 \\
\hline
\end{tabular}

From the above table 1, the comparison of ASS-TM with other criteria like short and long sentences, a system of baseline, WER, BLEU, and the model of IBM 2. Various types of sentences are chosen, and the output TL is compared for the efficiency rate achieved is $94.1 \%$. The results show a better translation performance for long sentences in the proposed approach using equation 5 , able 1 list statistical tests on the distribution of ASS-TM scores.

\subsection{Comparing SCG with accuracy performance}

Table 2: Accuracy analysis

\begin{tabular}{|c|c|c|c|c|}
\hline Methods & Accuracy & Precision & Recall & F1-score \\
\hline WBMT & 52.9 & 81.2 & 83.1 & 89.2 \\
\hline CPCS-HT & 69.1 & 72.1 & 76.5 & 84.3 \\
\hline PaCO-MT & 74.7 & 78.5 & 79.9 & 87.9 \\
\hline ERCA-AI & 61.3 & 68.8 & 73.8 & 79.5 \\
\hline SST-CL & 79.9 & 56.3 & 53.4 & 62.2 \\
\hline ASS-TM & 97.7 & 85.7 & 86.6 & 93.7 \\
\hline
\end{tabular}

As illustrated in table 2, the ASS-TM system generates 93.7percent of the human performance on F-1. Increased normalized performance compared to precision demonstrates the more comprehensive and integrated modeling approach employed in the TM system from the inclined equation 8.F-1 for different alternatives to lexical analysis with 3-5 best alternatives beyond which performance approximations have a meaningful increase in performance.

\subsection{Comparison of ASS-TM with other traditional methods}

Table 3 Comparison measures of ASS-TM 


\begin{tabular}{|c|c|c|c|c|c|c|}
\hline $\begin{array}{c}\text { Number } \\
\text { of } \\
\text { language } \\
\text { set }\end{array}$ & \multicolumn{6}{|c|}{ Comparison Ratio (\%) } \\
\cline { 2 - 7 } & WBMT & CPCS-HT & $\begin{array}{c}\text { PaCO- } \\
\text { MT }\end{array}$ & ERCA-AI & SST-CL & ASS-TM \\
\hline 10 & 37.0 & 40.1 & 56.1 & 50.2 & 71.0 & 73.1 \\
\hline 20 & 38.1 & 43.2 & 56.9 & 53.3 & 75.1 & 74.5 \\
\hline 30 & 39.4 & 46.3 & 57.0 & 55.5 & 78.3 & 76.7 \\
\hline 40 & 42.5 & 48.4 & 57.2 & 54.7 & 79.7 & 77.7 \\
\hline 50 & 44.4 & 50.8 & 57.3 & 57.9 & 81.6 & 88.9 \\
\hline 60 & 45.6 & 52.9 & 59.5 & 62.0 & 83.1 & 91.3 \\
\hline 70 & 49.7 & 51.1 & 62.7 & 64.3 & 82.3 & 92.6 \\
\hline 80 & 50.0 & 55.3 & 65.8 & 66.7 & 88.7 & 97.3 \\
\hline
\end{tabular}

From table 3 regarding the use of internet free access services, have most of them considered machine translation in language teaching is involved. The conclusion that parsing performance with SCFGs is closely linked to the translation rules explores the extension of high-quality translation rules. In many ways, an increased comparison is made with equation 10, with WBMT CPCS-HTPaCO-MT, ERCA-AI, SST-CL with ASS-TM. The graphical representation of ASS-TM comparison is given in a graphical representation as follows. 


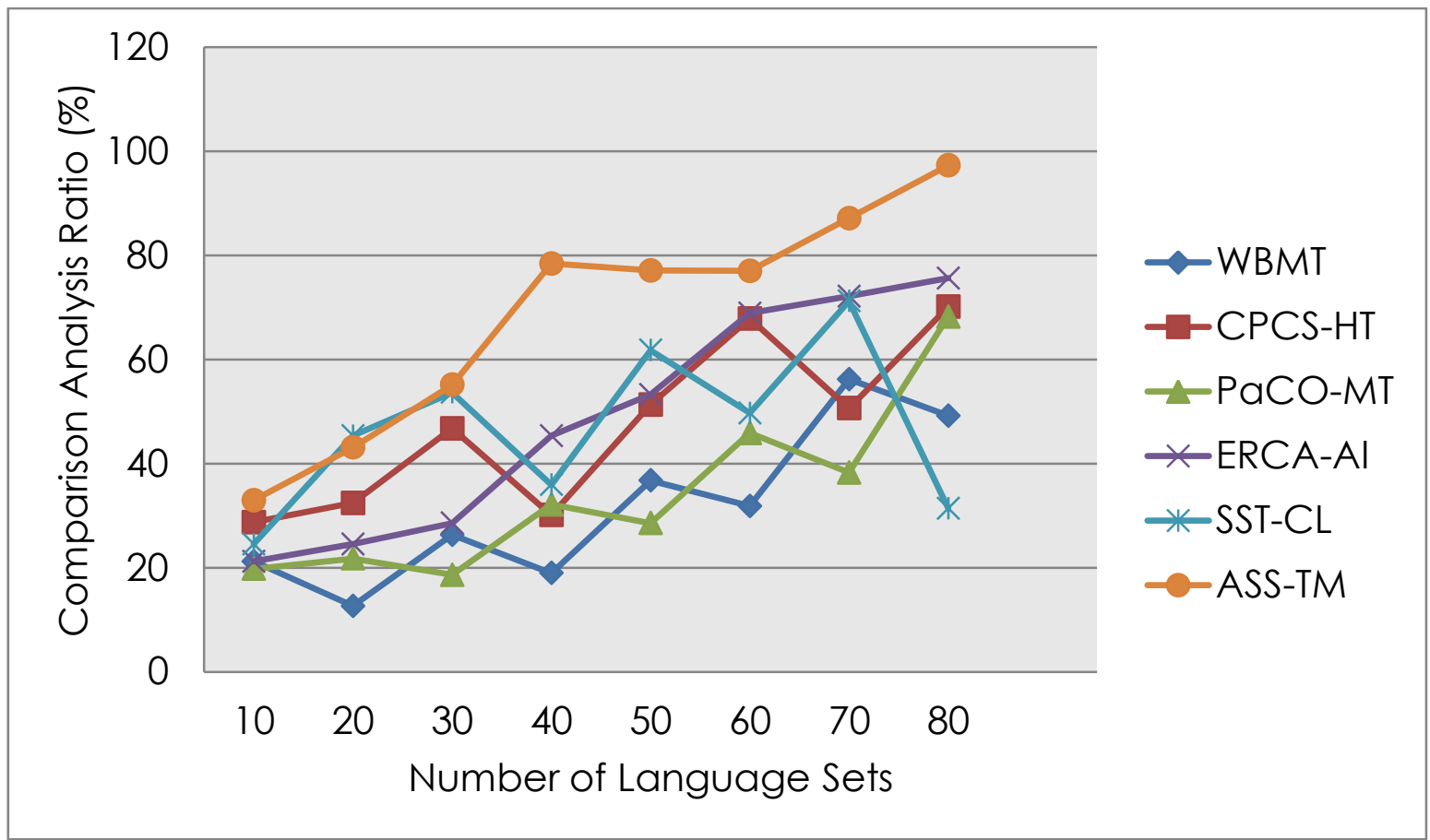

Figure 6 Comparison analysis

In addition to multilingual NMT, a further major research line seeks to bridge source and target languages through a vitally important language represented in figure 6. Corporate linguistic experiments show that our wordlevel sampling method can outperform content management methods and multilingual methods about translation quality and decoding efficiency from equation 13.

A highly efficient ASS-MT can record complete sentences, which gives a high-quality output to translating them. The text layout will be retained, and the translation returned almost immediately using our machine translation tool. The suggested ASS-MT concept is established to deal with the data shortage problem of small nonverbal communication translation.

\section{Conclusion}

The proposed technique ASS-TMprovides assumptions regarding the qualities and weaknesses of this innovation made concerning the concentration on access-free Internet-based translation services as the highest open type of translations, which are essentially appropriate for personal consumption and are lower for disseminating information objectives. The student's and translation instructor's observations, thinking, and 
perceptions about translation teaching in MT are involved. In general, both translation teachers and students used to see MT and free internet-based translation services in translation education as inventive learning and knowledge acquisition. However, future research can analyze the use of MT in translation classes through other common online/offline software and can compare translation memory implementation.

Combining a language approach with a mathematical approach allows the alignment and improvement of bi-lingual corporate processing from a technological standpoint to machine translation. The numerical simulation is based on the accuracy and effectiveness of the proposed method ASS-TM with $97.3 \%$ achievement.

\section{Ethics Declarations}

Conflict of interest

The authors declare that they have no conflict of interest.

Ethical approval

This article does not contain any studies with human participants or animals performed by any of the authors.

\section{References}

1. Billah, M. F. R. M., Saoda, N., Gao, J., \& Campbell, B. (2021, May). BLE Can See A Reinforcement Learning Approach for RF-based Indoor Occupancy Detection. In Proceedings of the 20thInternational Conference on Information Processing in Sensor Networks (co-located with CPS-IOT Week 2021) (pp. 132-147).

2. Do, D. T., Le, T. A., Nguyen, T. N., Li, X., \&Rabie, K. M. (2020). Joint impacts of imperfect CSI and imperfect SIC in cognitive radio-assisted NOMA-V2X communications. IEEE Access, 8, 128629-128645.

3. Subramaniyaswamy, V., Manogaran, G., Logesh, R., Vijayakumar, V., Chilamkurti, N., Malathi, D., \&Senthilselvan, N. (2019). An ontology-driven personalized food recommendation in loT-based healthcare system. The Journal of Supercomputing, 75(6), 3184-3216. 
4. Abd El-Latif, A. A., Abd-El-Atty, B., \&Venegas-Andraca, S. E. (2019). A novel image steganography technique based on quantum substitution boxes. Optics \& Laser Technology, 116, 92-102.

5. Javed, A. R., Rehman, S. U., Khan, M. U., Alazab, M., \& Khan, H. U. (2021). Betalogger: Smartphone Sensor-based Side-channel Attack Detection and Text Inference Using Language Modeling and Dense MultiLayer Neural Network. Transactions on Asian and Low-Resource Languagelnformation Processing, 20(5), 1-17.

6. Elhoseny, M., Shankar, K., Lakshmanaprabu, S. K., Maseleno, A., \&Arunkumar, N. (2020). Hybrid optimization with cryptography encryption for medical image security in Internet of Things. Neural computing and applications, 32(15), 10979-10993.

7. Amudha, G., \&Narayanasamy, P. (2018). Distributed location and trustbased replica detection in wireless sensor networks. Wireless Personal Communications, 102(4), 3303-3321.

8. Gao, J., Wang, H., \&Shen, H. (2020, May). Smartly handling renewable energy instability in supporting a cloud datacenter. In 2020 IEEE international parallel and distributed processing symposium (IPDPS) (pP. 769-778). IEEE.

9. Shakeel, P. M., Baskar, S., Fouad, H., Manogaran, G., Saravanan, V., \&Xin, Q. (2020). Creating Collision-Free Communication in IOT with 6G Using Multiple Machine Access Learning Collision Avoidance Protocol. Mobile Networks and Applications, 1-12.

10.Le, N. T., Wang, J. W., Wang, C. C., \& Nguyen, T. N. (2019). Automatic defect inspection for coated eyeglass based on symmetrized energy analysis of color channels. Symmetry, 11(12), 1518.

11.Kumar, P. M., Gandhi, U., Varatharajan, R., Manogaran, G., Jidhesh, R., \&Vadivel, T. (2019). Intelligent face recognition and navigation system using neural learning for smart security in Internet of Things. Cluster Computing, 22(4), 7733-7744. 
12. Abou-Nassar, E. M., Iliyasu, A. M., El-Kafrawy, P. M., Song, O. Y., Bashir, A. K., \&Abd El-Latif, A. A. (2020). DITrust chain: towards blockchain-based trust models for sustainable healthcare IOT systems. IEEE Access, 8, 111223 111238.

13. Amudha, G. (2021). Dilated Transaction Access and Retrieval: Improving the Information Retrieval of Blockchain-Assimilated Internet of Things Transactions. Wireless Personal Communications, 1-21.

14. Elhoseny, M., Tharwat, A., Farouk, A., \&Hassanien, A. E. (2017). K-coverage model based on genetic algorithm to extend WSN lifetime. IEEE sensors letters, 1(4), 1-4.

15. Kuthadi, V. M., Selvaraj, R., Baskar, S., Shakeel, P. M., \&Ranjan, A. (2021). Optimized Energy Management Model on Data Distributing Framework of Wireless Sensor Network in loT System. Wireless Personal Communications, 1-27. https://doi.org/10.1007/s1 1277-021-08583-0.

16.Xu, C., Qin, T., Wang, G., \& Liu, T. Y. (2019). Polygon-Net: A General Framework for Jointly Boosting Multiple Unsupervised Neural Machine Translation Models. In IJCAI (pp. 5320-5326).

17.Sabtan, Y. M. N., \& Salalah, O. (2020). Teaching Arabic machine translation to EFL student translators: A case study of Omani translation undergraduates. International Journal of English Linguistics, 10(2), 184.

18. Kim, S. (2019). Playing with machine translation in the language classroom: Affordances and constraints. Multimedia-Assisted Language Learning, 22(2), 9-28.

19. Al-Shedivat, M., \& Parikh, A. P. (2019). Consistency by agreement in zeroshot neural machine translation. arXiv preprint arXiv:1904.02338.

20. Xu, T., Ozbek, O. I., Marks, S., Korrapati, S., \&Ahmadnia, B. (2020). SpanishTurkish Low-Resource Machine Translation: Unsupervised Learning vs. Round-Tripping. American Journal of Artificial Intelligence, 4(2), 42-49.

21. Way, A. (2020). Machine translation: where are we at today?. The Bloomsbury companion to language industry studies, 31 1-332. 
22. Alfuraih, R. F. (2020). The undergraduate learner translator corpus: a new resource for translation studies and computational linguistics. Language Resources and Evaluation, 54(3), 801-830.

23. Yang, Y., Wang, X., \& Yuan, Q. (2021). Measuring the usability of machine translation in the classroom context. Translation and Interpreting Studies, 16(1), 101-123.

24. Vardaro, J., Schaeffer, M., \& Hansen-Schirra, S. (2019, September). Translation quality and error recognition in professional neural machine translation post-editing. In Informatics (Vol. 6, No. 3, p. 41). Multidisciplinary Digital Publishing Institute.

25.Montoya, H. E. G., Rojas, K. D. R., \&Oncevay, A. (2019, August). A continuous improvement framework of machine translation for Shipibokonibo. In Proceedings of the 2nd Workshop on Technologies for MT of Low Resource Languages (pp. 17-23).

26. Wu, J., Wang, X., \& Wang, W. Y. (2019). Extract and edit: An alternative to back-translation for unsupervised neural machine translation. arXiv preprint arXiv: 1904.02331.

27. Way, A., Hasanuzzaman, M., \&Haque, R. (2019). Multiword units in machine translation and translation technology--RuslanMitkov, Johanna Monti, Gloria Corpas Pastor, and VioletaSeretan (eds), Book Review. Machine Translation, 341, 1-6.

28. Kahlon, N. K., \& Singh, W. (2021). Machine translation from text to sign language: a systematic review. Universal Access in the Information Society, 1-35.

29. Aksoy, B. N. (2019). Technology-based innovations in the teaching of translation: an insight into whys and hows. AnaleleUniversitățiiOvidius din Constanța. SeriaFilologie, 30(1), 191-199.

30.Luqman, H., \& Mahmoud, S. A. (2019). A machine translation system from Arabic sign language to Arabic. Universal Access in the Information Society, 1-14. 